ARTICLE

Received 6 Sep 2012 | Accepted 12 Dec 2012 | Published 29 Jan 2013 | Updated 12 Dec 2013

DOI: $10.1038 /$ ncomms2380

\title{
The impact of free-ranging domestic cats on wildlife of the United States
}

Scott R. Loss' ${ }^{1}$ Tom Will ${ }^{2} \&$ Peter P. Marra ${ }^{1}$

Anthropogenic threats, such as collisions with man-made structures, vehicles, poisoning and predation by domestic pets, combine to kill billions of wildlife annually. Free-ranging domestic cats have been introduced globally and have contributed to multiple wildlife extinctions on islands. The magnitude of mortality they cause in mainland areas remains speculative, with large-scale estimates based on non-systematic analyses and little consideration of scientific data. Here we conduct a systematic review and quantitatively estimate mortality caused by cats in the United States. We estimate that free-ranging domestic cats kill 1.3-4.0 billion birds and 6.3-22.3 billion mammals annually. Un-owned cats, as opposed to owned pets, cause the majority of this mortality. Our findings suggest that free-ranging cats cause substantially greater wildlife mortality than previously thought and are likely the single greatest source of anthropogenic mortality for US birds and mammals. Scientifically sound conservation and policy intervention is needed to reduce this impact.

\footnotetext{
${ }^{1}$ Migratory Bird Center, Smithsonian Conservation Biology Institute, National Zoological Park, P.O. Box 37012 MRC 5503, Washington, District of Columbia 20013, USA. ${ }^{2}$ U.S. Fish and Wildlife Service, Division of Migratory Birds, Midwest Regional Office, 3815 American Boulevard East, Bloomington, Minnesota 20013, USA. Correspondence and requests for materials should be addressed to S.R.L. (email: LossS@si.edu).
} 
D omestic cats (Felis catus) are predators that humans have introduced globally ${ }^{1,2}$ and that have been listed among the 100 worst non-native invasive species in the world ${ }^{3}$. Free-ranging cats on islands have caused or contributed to 33 (14\%) of the modern bird, mammal and reptile extinctions recorded by the International Union for Conservation of Nature (IUCN) Red List ${ }^{4}$. Mounting evidence from three continents indicates that cats can also locally reduce mainland bird and mammal populations ${ }^{5-7}$ and cause a substantial proportion of total wildlife mortality ${ }^{8-10}$. Despite these harmful effects, policies for management of free-ranging cat populations and regulation of pet ownership behaviours are dictated by animal welfare issues rather than ecological impacts ${ }^{11}$. Projects to manage free-ranging cats, such as Trap-Neuter-Return (TNR) colonies, are potentially harmful to wildlife populations, but are implemented across the United States without widespread public knowledge, consideration of scientific evidence or the environmental review processes typically required for actions with harmful environmental consequences ${ }^{11,12}$.

A major reason for the current non-scientific approach to management of free-ranging cats is that total mortality from cat predation is often argued to be negligible compared with other anthropogenic threats, such as collisions with man-made structures and habitat destruction. However, assessing the conservation importance of a mortality source requires identification of which species are being killed (for example, native versus non-native invasive species and rare versus common species) in addition to estimation of total numbers of fatalities. Estimates of annual US bird mortality from predation by all cats, including both owned and un-owned cats, are in the hundreds of millions ${ }^{13,14}$ (we define un-owned cats to include farm/barn cats, strays that are fed by humans but not granted access to habitations, cats in subsidized colonies and cats that are completely feral). This magnitude would place cats among the top sources of anthropogenic bird mortality; however, window and building collisions have been suggested to cause even greater mortality ${ }^{15-17}$. Existing estimates of mortality from cat predation are speculative and not based on scientific data ${ }^{13-16}$ or, at best, are based on extrapolation of results from a single study ${ }^{18}$. In addition, no large-scale mortality estimates exist for mammals, which form a substantial component of cat diets.

We conducted a data-driven systematic review of studies that estimate predation rates of owned and un-owned cats, and estimated the magnitude of bird and mammal mortality caused by all cats across the contiguous United States (all states excluding Alaska and Hawaii). We estimate that free-ranging domestic cats kill 1.3-4.0 billion birds and 6.3-22.3 billion mammals annually, and that un-owned cats cause the majority of this mortality. This magnitude of mortality is far greater than previous estimates of cat predation on wildlife and may exceed all other sources of anthropogenic mortality of US birds and mammals.

\section{Results}

The magnitude of bird mortality caused by cat predation. After excluding studies that did not meet a priori inclusion criteria designed to increase the accuracy of our analysis, we developed probability distributions of predation rates on birds and mammals. We combined predation rate distributions with literaturederived probability distributions for US cat population sizes, and we also accounted for the proportion of owned cats allowed outdoors, the proportion of owned and un-owned cats that hunt, and imperfect detection of owned cats' prey items.

We generated an estimated range of bird and mammal mortality caused by cat predation by incorporating the above distributions-including separate predation rate distributions for owned and un-owned cats-and running 10,000 calculation iterations. We augmented US predation data by incorporating predation rate estimates from other temperate regions (Supplementary Table S1). For birds, we generated three US mortality estimates based on predation data from studies in: (1) the United States, (2) the United States and Europe and (3) the United States, Europe, and other temperate regions (primarily Australia and New Zealand). Owing to a lack of US studies of un-owned cat predation on mammals, we estimated mammal mortality using data groupings 2 and 3 . We based all other probability distributions on US studies (distribution details in Table 1; data in Supplementary Table S2).

The three estimates of bird mortality varied moderately, with a $19 \%$ difference among median estimates (Table 2). We focus interpretation on the estimate generated using US and European predation data because it is the lowest value. Furthermore, this estimate is more likely to be representative of the US than the estimate based on incorporation of data from Australia and New Zealand, where the wildlife fauna and climate are less similar to the United States. We estimate that cats in the contiguous United States annually kill between 1.3 and 4.0 billion birds (median $=2.4$ billion) (Fig. 1a), with $\sim 69 \%$ of this mortality caused by un-owned cats. The predation estimate for un-owned cats was higher primarily due to predation rates by this group averaging three times greater than rates for owned cats.

The magnitude of mammal mortality caused by cat predation. Our estimate of mammal mortality was robust to the choice of predation data as evidenced by a $1.6 \%$ difference between the two median estimates (Table 2). We focus interpretation on the lower estimate, which was based on United States and European predation data and US values of other parameters. We estimate annual mammal mortality in the contiguous United States at between 6.3 and 22.3 billion (median $=12.3$ billion) (Fig. $1 \mathrm{~b}$ ) with $89 \%$ of this mortality caused by un-owned cats. The estimate that incorporated European data (but not data from Australia and New Zealand) may be slightly lower because wildlife across much of Europe were historically exposed to predation by a similarlysized wild cat (Felis sylvestris) and, therefore, may be less naive to predation by domestic cats. However, it is unlikely that European wildlife have fully adapted to the unusually high densities of domestic cats in much of this continent ${ }^{9}$.

Factors explaining estimate uncertainty. For both birds and mammals, sensitivity analyses indicated that un-owned cat parameters explained the greatest variation in total mortality estimates (Fig. 2). Un-owned cat population size explained the greatest variation in mortality estimates ( $42 \%$ for birds and $51 \%$ for mammals), and the un-owned cat predation rate explained the second greatest variation (24\% for birds and $40 \%$ for mammals). The only other parameters that explained $>5 \%$ of variation in mortality estimates were the owned cat predation rate on birds (16\%) and the correction factor for imperfect detection of owned cats' prey items $(8 \%)$.

\section{Discussion}

Our estimate of bird mortality far exceeds any previously estimated US figure for cats ${ }^{13,14,16}$, as well as estimates for any other direct source of anthropogenic mortality, including collisions with windows, buildings, communication towers, vehicles and pesticide poisoning ${ }^{13,15-21}$. Systematic reviews like ours, which includes protocol formulation, a data search strategy, data inclusion criteria, data extraction and formal quantitative analyses $^{22}$, are scarce for other anthropogenic mortality sources. ${ }^{21}$ Increased rigour of mortality estimates should be a high priority 
Table 1 | Probability distributions used for parameters in cat predation model.

\section{Parameter}

Owned cats

Number of owned cats in contiguous United States

Proportion of owned cats with outdoor access

Proportion of outdoor owned cats that hunt

Correction for owned cats not returning prey

$B I R D$ return rate per cat per year

US studies

US and Europe studies

All temperate studies

MAMMAL return rate per cat per year

US studies

US and Europe studies

All temperate studies

REPTILE return rate per cat per year

US studies

US and Europe studies

All temperate studies

AMPHIBIAN return rate per cat per year

US studies

US and Europe studies

All temperate studies

Un-owned cats

Number of un-owned cats in contiguous United States

Proportion of un-owned cats that hunt

BIRD predation rate per cat per year

US studies

US and Europe studies

All temperate studies

MAMMAL predation rate per cat per year

US studies

US and Europe studies

All temperate studies

REPTILE predation rate per cat per year

US studies

US and Europe studies

All temperate studies

AMPHIBIAN predation rate per cat per year

US studies

US and Europe studies

All temperate studies

Number of studies used $\star$

2

8

3

4

11

17

1

7

0

8

0

1

5

5

2

8

11

19

6

7

13

1

2

10
Distribution type

Normal
Uniform
Uniform
Uniform

Uniform

Uniform

Uniform

$\mathrm{NA \dagger}$

Uniform

Uniform

NA

NA

Uniform

NA

NA

Uniform

Uniform

Uniform

Uniform

Uniform

Uniform

Uniform

Uniform

Uniform
Distribution parameters

Mean $=84 M$, s.d. $=2.5 \mathrm{M}$
$\operatorname{Min}=0.4, \max =0.7$
$\operatorname{Min}=0.5, \max =0.8$
$\operatorname{Min}=1.2, \max =3.3$

Min $=1.0, \max =34.1$

$\operatorname{Min}=4.2, \max =18.3$

$\operatorname{Min}=3.4, \max =13.2$

NA

$\operatorname{Min}=11.1, \max =29.5$

$\operatorname{Min}=8.7, \max =21.8$

NA

NA

$\operatorname{Min}=0.4, \max =2.21$

NA
NA
Min $=0.05, \max =0.5$

$\operatorname{Min}=30 \mathrm{M}, \max =80 \mathrm{M}$

$\operatorname{Min}=0.8, \max =1.0$

Min $=24.4, \max =51.4$

$\operatorname{Min}=23.2, \max =46.2$

Min $=30.0, \max =47.6$

$\operatorname{Min}=162.3, \max =354.9$

Min $=139.4, \max =328.6$

$\operatorname{Min}=177.3, \max =299.5$

*Number of studies found that include an estimate of the model parameter.

$\dagger$ No calculation was conducted for this data grouping because of limited data.

Table 2 | Median estimates of annual wildlife mortality caused by cat predation in the contiguous United States.

\begin{tabular}{|c|c|c|c|c|}
\hline & \multirow[t]{2}{*}{ Predation data used } & \multicolumn{3}{|c|}{ Mortality estimate (millions) } \\
\hline & & Owned cats & Un-owned cats & Total \\
\hline \multirow[t]{3}{*}{ Bird } & US & $1,053(104-3,039)^{\star}$ & $1,792(861-3,276)$ & $2,967(1,358-5,324)$ \\
\hline & US and Europe & $684(221-1,682) \dagger$ & $1,652(803-2,955)$ & $2,407(1,306-3,992)$ \\
\hline & All temperate & $508(172-1,226)$ & $1,876(983-3,124)$ & $2,437(1,378-3,814)$ \\
\hline \multirow[t]{2}{*}{ Mammal } & US and Europe & $1,249(512-2,862)$ & $10,903(4,991-20,874)$ & $12,269(6,259-22,257)$ \\
\hline & All temperate & $958(397-2,117)$ & $11,426(5,874-19,451)$ & $12,473(6,874-20,421)$ \\
\hline Both Taxa & & $1,933(733-4,544)$ & $12,555(5,794-23,829)$ & $14,676(7,565-26,249)$ \\
\hline
\end{tabular}


and will allow increased comparability of mortality sources ${ }^{23}$. Nonetheless, no estimates of any other anthropogenic mortality source approach the value we calculated for cat predation, and our estimate is the first for cats to be based on rigorous datadriven methods. Notably, we excluded high local predation rates and used assumptions that led to minimum predation rate estimates for un-owned cats; therefore, actual numbers of birds killed may be even greater than our estimates.

Free-roaming cats in the United States may also have a substantial impact on reptiles and amphibians. However, US studies of cat predation on these taxa are scarce. To generate a first approximation of US predation rates on reptiles and amphibians, we used the same model of cat predation along with estimates of cat predation rates on these taxa from studies in Europe, Australia and New Zealand. We estimate that between
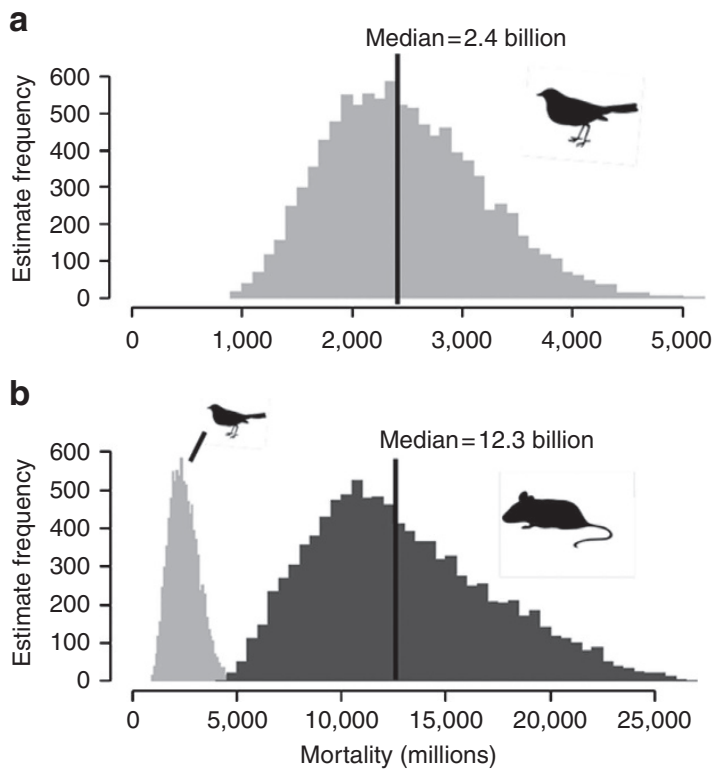

Figure 1 | Estimates of cat predation on US birds and mammals.

(a) Probability distribution of estimated bird mortality caused by all freeranging cats in mainland areas of the contiguous United States.

(b) Probability distribution of estimated mammal mortality caused by all free-ranging cats in mainland areas of the contiguous United States.
228 to 871 million reptiles (median $=478$ million) and between 86 and 320 million amphibians (median $=173$ million) could be killed by cats in the contiguous United States each year. Reptile and amphibian populations, and, therefore, cat predation rates, may differ between the regions where we gathered predation data for these taxa and the United States. Furthermore, reptiles and amphibians are unavailable as prey during winter across much of the United States. Additional research is needed to clarify impacts of cats on US herpetofauna, especially given numerous anthropogenic stressors that threaten their populations (for example, climate change, habitat loss and infectious diseases) and documented extinctions of reptiles and amphibians due to cat predation in other regions ${ }^{4,24}$.

The exceptionally high estimate of mammal mortality from cat predation is supported by individual US studies that illustrate high annual predation rates by individual un-owned cats in excess of 200 mammals per year ${ }^{6,25-28}$ and the consistent finding that cats preferentially depredate mammals over other taxa (Supplementary Table S1). Even with a lower yearly predation rate of 100 mammals per cat, annual mortality would range from 3-8 billion mammals just for un-owned cats, based on a population estimate of between 30 and 80 million un-owned cats. This estimated level of mortality could exceed any other direct source of anthropogenic mortality for small mammals; however, we are unaware of studies that have systematically quantified direct anthropogenic mortality of small terrestrial mammals across large scales.

Native species make up the majority of the birds preyed upon by cats. On average, only $33 \%$ of bird prey items identified to species were non-native species in 10 studies with 438 specimens of 58 species (Supplementary Table S3). For mammals, patterns of predation on native and non-native species are less clear and appear to vary by landscape type. In densely populated urban areas where native small mammals are less common, non-native species of rats and mice can make up a substantial component of mammalian prey $^{29}$. However, studies of mammals in suburban and rural areas found that $75-100 \%$ of mammalian prey were native mice, shrews, voles, squirrels and rabbits ${ }^{26,30,31}$. Further research of mammals is needed to clarify patterns of predation by both owned and un-owned cats on native and non-native mammals, and across different landscape types.

Sensitivity analyses indicate that additional research of un-owned cats will continue to improve precision of mortality estimates. Our finding that un-owned cat population size and

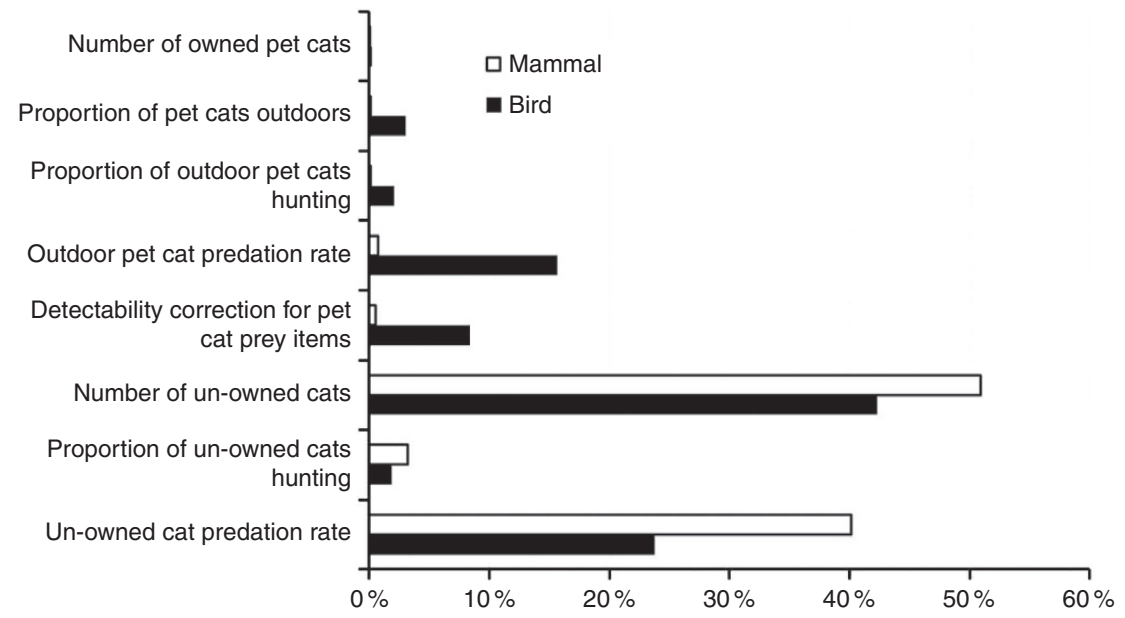

Figure 2 | Factors explaining uncertainty in estimates of wildlife mortality from cat predation. Amount of variation in estimates of wildlife mortality in the contiguous United States contributed by each parameter in the cat predation model (percentages represent adjusted $R^{2}$ values from multiple regression models). 
predation rate explained the greatest variation in mortality estimates reflects the current lack of knowledge about un-owned cats. No precise estimate of the un-owned cat population exists for the United States because obtaining such an estimate is cost prohibitive, and feral un-owned cats are wary of humans and tend to be solitary outside of urban areas. In addition, human subsidized colonies of un-owned cats are maintained without widespread public knowledge. For example, in Washington DC alone there are $>300$ managed colonies of un-owned cats and an unknown number of unmanaged colonies. Population size estimates can be improved by incorporating observations of free-ranging cats into a wildlife mortality reporting database $\mathrm{e}^{23}$.

Context for the population impact of a mortality source depends on comparing mortality estimates to estimates of population abundance of individual species. However, continental-scale estimates of wildlife population abundance are uncertain due to spatio-temporal variation in numbers. For mammals, clarification of the population impacts of cat predation is hindered by the absence of nationwide population estimates. For all North American land birds, the group of species most susceptible to mainland cat predation (Supplementary Table S3), existing estimates range from 10-20 billion individuals in North America $^{32}$. A lack of detail about relative proportions of different bird species killed by cats and spatio-temporal variation of these proportions makes it difficult to identify the species and populations that are most vulnerable. The magnitude of our mortality estimates suggest that cats are likely causing population declines for some species and in some regions. Threatened and endangered wildlife species on islands are most susceptible to the effects of cat predation, and this may also be true for vulnerable species in localized mainland areas ${ }^{5}$ because small numbers of fatalities could cause significant population declines. Threatened species in close proximity to cat colonies-including managed TNR colonies ${ }^{11,12}$-face an especially high level of risk; therefore, cat colonies in such locations comprise a wildlife management priority. Claims that TNR colonies are effective in reducing cat populations, and, therefore, wildlife mortality, are not supported by peer-reviewed scientific studies ${ }^{11}$.

Our estimates should alert policy makers and the general public about the large magnitude of wildlife mortality caused by free-ranging cats. Structured decisions about actions to reduce wildlife mortality require a quantitative evidence base. We provide evidence of large-scale cat predation impacts based on systematic analysis of multiple data sources. Future specific management decisions, both in the United States and globally, must be further informed by fine scale research that allows analysis of population responses to cats and assessment of the success of particular management actions. We are not suggesting that other anthropogenic threats that kill fewer individuals are biologically unimportant. Virtually nothing is known about the cumulative population impacts of multiple mortality sources. Furthermore, comparison of total mortality numbers has limited use for prioritization of risks and development of conservation objectives. Combining per species estimates of mortality with population size estimates will provide the greatest information about the risk of population-level impacts of cat predation. Although our results suggest that owned cats have relatively less impact than un-owned cats, owned cats still cause substantial wildlife mortality (Table 2); simple solutions to reduce mortality caused by pets, such as limiting or preventing outdoor access, should be pursued. Efforts to better quantify and minimize mortality from all anthropogenic threats are needed to increase sustainability of wildlife populations.

The magnitude of wildlife mortality caused by cats that we report here far exceeds all prior estimates. Available evidence suggests that mortality from cat predation is likely to be substantial in all parts of the world where free-ranging cats occur. This mortality is of particular concern within the context of steadily increasing populations of owned cats, the potential for increasing populations of un-owned cats ${ }^{12}$, and an increasing abundance of direct and indirect mortality sources that threaten wildlife in the United States and globally.

\section{Methods}

Literature search. We searched JSTOR, Google Scholar, and the Web of Science database (formerly ISI Web of Science) within the Web of Knowledge search engine published by Thomson Reuters to identify studies that document cat predation on birds and mammals. We initially focused this search on US studies, but due to a limited sample of these studies, we expanded the search to include predation research from other temperate regions. We also searched for studies providing estimates of cat population sizes at the scale of the contiguous United States and for US studies that estimate the proportion of owned cats with outdoor access and the proportion of cats that hunt wildlife. The search terms we used included: 'domestic cat' in combination with 'predation,' 'prey,' 'diet,' 'food item' and 'mortality'; all previous terms with 'domestic cat' replaced by 'Felis catus,' 'feral,' 'stray,' 'farm,' 'free-ranging,' and 'pet'; 'trap-neuter-return colony'; 'TNR colony'; and 'cat predation' in combination with 'wildlife,' 'bird,' 'mammal,' and 'rodent'. We checked reference lists of articles to identify additional relevant studies. Lead authors of three studies were also contacted to enquire whether they knew of ongoing or completed unpublished studies of cat predation in the United States.

Classification of cat ranging behaviour. We grouped studies based on the ranging behaviour of cats investigated. We defined owned cats to include owned cats in both rural and urban areas that spend at least some time indoors and are also granted outdoor access. We defined un-owned cats to include all un-owned cats that spend all of their time outdoors. The un-owned cat group includes semi-feral cats that are sometimes considered pets (for example, farm/barn cats and strays that are fed by humans but not granted access to habitations), cats in subsidized (including TNR) colonies, and cats that are completely feral (that is, completely independent and rarely interacting with humans). We did not classify cats by landscape type or whether they receive food from humans because the amount of time cats spend outdoors is a major determinant of predation rates ${ }^{33,34}$ and because predation is independent of whether cats are fed by humans $s^{6,34,35}$.

Study inclusion criteria. Studies were only included if: (1) they clearly reported cat ranging behaviour (that is, a description of whether cats were owned or un-owned and whether they were outdoor cats or indoor-outdoor cats), and (2) the group of cats investigated fit exclusively into one of the two groups we defined above (that is, we excluded studies that lumped owned and un-owned cats in a single predation rate estimate). For some studies, we extracted a portion of data that met these criteria but excluded other data from cats with unknown ranging behaviour. We only included mainland and large island (New Zealand and United Kingdom) predation studies, because cat predation on small islands is often exceptionally high $^{36,37}$ and focused on colony nesting seabirds ${ }^{38}$. We excluded studies from outside temperate regions and those with predation rate estimates based on fewer than 10 cats, $<1$ month of sampling, or on cats that were experimentally manipulated (for example, by fitting them with bells or behaviour altering bibs). We included studies that used cat owners' records of prey returns, but we excluded those that asked owners to estimate past prey returns because such questionnaires may lead to bias in estimation of predation rates ${ }^{39}$. (For a list of all included and excluded studies, see Supplementary Table S1).

Data extraction and standardization of predation rates. Most studies report an estimate of cat predation rate (that is, daily, monthly or annual prey killed per cat) or present data that allowed us to calculate this rate. When studies only reported predation rate estimates for all wildlife combined, we calculated separate predation rates by extracting taxa-specific prey counts from tables or figures and multiplying the total predation rate by the proportion of prey items in each taxon. If taxaspecific counts were not provided, we directly contacted authors to obtain this information. For studies that presented low, medium and high estimates or low and high estimates, we used the medium and average values, respectively. For studies that presented more than one predation estimate for cats with similar ranging behaviour (for example, owned cats in rural and urban areas), we calculated the average predation rate.

Nearly all studies of un-owned cats report numbers or frequencies of occurrence of different taxa in stomachs and/or scats. For studies reporting numbers of prey items, we estimated annual predation rates by assuming one stomach or scat sample represented a cat's average daily prey intake (for example, an average of one prey item per stomach or scat $=365$ prey per cat per year). This assumption likely resulted in conservative estimates because cats generally digest prey within $12 \mathrm{~h}$ (ref. 28) and can produce two or more scats each day ${ }^{29}$. For studies reporting occurrence frequencies of prey items, we assumed this proportion represented a cat's average daily prey intake (for example, a $10 \%$ bird occurrence rate $=0.1$ bird 
per stomach or scat $=36.5$ birds per cat per year). This assumption results in coarse predation rate estimates, but estimates from this approach are even more conservative than those from the first assumption because many stomachs and scats undoubtedly included more than one bird or mammal.

Predation rate estimates from many studies were based on continuous yearround sampling or multiple sampling occasions covering all seasons. However, seasonal coverage of some studies was incomplete. To generate full-year predation rate estimates in these cases, we adjusted partial-year predation estimates according to the average proportion of prey taken in each month as determined from yearround studies reporting monthly data (birds and mammals ${ }^{8,33}$, birds only ${ }^{7,40}$ ). For partial-year estimates from the northern hemisphere, we offset monthly estimates from southern hemisphere studies by 6 months. The final annual predation rate estimates for all studies are presented in Supplementary Table S1. The year-round studies we used represent different geographical regions (for birds-England, Kansas (US), Australia and New Zealand; for mammals-England and Australia) with varying climates and slightly varying seasonal patterns of predation. For both birds and mammals, averaging across full-year studies resulted in higher proportions of predation in the spring and summer compared with fall and winter, an expected pattern for much of the United States. The reference studies we used, therefore, provide a reasonable baseline for correcting to full-year mortality estimates. This approach greatly improves upon the assumption that mortality is negligible during the period of the year not covered by sampling.

Quantification of annual mortality from cat predation. We estimated wildlife mortality in the contiguous United States by multiplying data-derived probability distributions of predation rates by distributions of estimated cat abundance, following ${ }^{41}$. Quantification was conducted separately for owned and un-owned cats and for birds and mammals. As there was a relatively small sample of US studies that estimated predation rates ( $n=14$ and 10 for birds and mammals, respectively), we repeated calculations using predation rate distributions that were augmented with predation rates from Europe and all temperate zones. However, we only used studies from the contiguous United States to construct all other probability distributions (listed below).

We estimated mortality using the following model of cat predation:

Annual mortality from owned cats $(\mathrm{mp})=\mathrm{npc} \times \operatorname{pod} \times \operatorname{pph} \times \operatorname{ppr} \times$ cor

Annual mortality from unowned cats $(\mathrm{mf})=\mathrm{nfc} \times \mathrm{pfh} \times \mathrm{fpr}$

Total annual mortality from all cats $=\mathrm{mp}+\mathrm{mf}$

where npc is the number of owned cats in the contiguous United States, pod is the proportion of owned cats granted outdoor access, pph is the proportion of outdoor owned cats that hunt wildlife, ppr is the annual predation rate by owned cats, cor is a correction factor to account for owned cats not returning all prey to owners, nfc is the number of un-owned cats in the contiguous United States, pfh is the proportion of un-owned cats that hunt wildlife, and fpr is the annual predation rate by un-owned cats. From the probability distribution of each parameter (see Table 1 and Supplementary Methods for details about the specific probability distributions used), we randomly drew one value and used the above formulas to calculate mortality. Random draws were made using distribution functions in Programme R (rnorm and runif commands for normal and uniform distributions, respectively). We conducted 10,000 random draws to estimate a potential range of annual predation on each wildlife taxa. For all analyses, we report median mortality estimates and lower and upper estimates bracketing the central $95 \%$ of values.

Sensitivity analyses. We used multiple linear regression analysis to assess how much variance in mortality estimates was explained by the probability distribution for each parameter. We treated total mortality estimates as the dependent variable $(n=10,000)$ and we defined a predictor variable for each parameter that consisted of the 10,000 randomly drawn values. We used adjusted $R^{2}$ values to interpret the percentage of variance explained by each parameter.

\section{References}

1. Baker, P. J., Soulsbury, C. D., Iossa, G. \& Harris, S. in Urban Carnivores. (eds Gehrt, S. D., Riley, S. P. D. \& Cypher, B. L.) 157-171 (John Hopkins University Press, 2010)

2. Fitzgerald, B. J. in The Domestic Cat: The Biology of its Behaviour. (eds Turner, D. C. \& Bateson, P.) 123-150 (Cambridge University Press, 1990).

3. Lowe, S., Browne, M. \& Boudjelas, S. 100 of the World's Worst Invasive Alien Species: a Selection from The Global Invasive Species Database (Invasive Species Specialist Group, International Union for Conservation of Nature, 2000).

4. Medina, F. M. et al. A global review of the impacts of invasive cats on island endangered vertebrates. Global Change Biol. 17, 3503-3510 (2011).

5. Crooks, K. R. \& Soule, M. E. Mesopredator release and avifaunal extinctions in a fragmented system. Nature 400, 563-566 (1999).
6. Hawkins, C. C., Grant, W. E. \& Longnecker, M. T. in Proceedings of the 4th International Urban Wildlife Symposium. (eds Shaw, W. W., Harris, L. K. \& Vandruff, L.) 164-170 (University of Arizona, Tucson, AZ, 2004).

7. van Heezik, Y., Smyth, A., Adams, A. \& Gordon, J. Do domestic cats impose an unsustainable harvest on urban bird populations? Biol. Conserv. 143, 121-130 (2010).

8. Churcher, P. B. \& Lawton, J. H. Predation by domestic cats in an English village. J. Zool. London 212, 439-455 (1987).

9. Baker, P. J., Molony, S., Stone, E., Cuthill, I. C. \& Harris, S. Cats about town: is predation by free-ranging pet cats (Felis catus) likely to affect urban bird populations? IBIS 150(Suppl. 1): 86-99 (2008).

10. Balogh, A. L., Ryder, T. B. \& Marra, P. P. Population demography of Gray Catbirds in the suburban matrix: sources, sinks, and domestic cats. J. Ornitholol. 152, 717-726 (2011).

11. Longcore, T., Rich, C. \& Sullivan, L. M. Critical assessment of claims regarding management of feral cats by trap-neuter-return. Conserv. Biol. 23, 887-894 (2009).

12. Lepczyk, C. A. et al. What conservation biologists can do to counter trap-neuter-return: response to Longcore et al.. Conserv. Biol. 24, 627-629 (2010).

13. Gill, F. B. Ornithology, 2nd edn. (W.H. Freeman Publishers, 1994).

14. Dauphiné, N. \& Cooper, R. J. Impacts of free-ranging domestic cats (Felis catus) on birds in the United States: a review of recent research with conservation and management recommendations. Proceedings of the Fourth International Partners in Flight Conference: Tundra to Tropics 205-219 (Partners in Flight, US, 2009).

15. Banks, R. C. Human related mortality of birds in the United States. Special Scientific Report-Wildlife N. 215 (US Dept. of the Interior-Fish and Wildlife Service, 1979).

16. Erickson, W. P., Johnson, G. D. \& Young, Jr D. P. A summary and comparison of bird mortality from anthropogenic causes with an emphasis on collisions. Tech. Rep PSW-GTR-191 1029-1042 (US Dept. of Agriculture-Forest Service, 2005).

17. Klem, Jr D. Avian mortality at windows: the second largest human source of bird mortality on earth. Proceedings of the Fourth International Partners in Flight Conference: Tundra to Tropics 244-251 (Partners in Flight, 2009).

18. Coleman, J. S. \& Temple, S. A. On the Prowl. Wisconsin Nat. Res. Mag. (1996).

19. Pimentel, D., Greiner, A. \& Bashore, T. Economic and environmental costs of pesticide use. Arch. Environ. Con. Tox. 21, 84-90 (1991).

20. Manville, II A. Towers, turbines, power lines, and buildings-steps being taken by the U.S. Fish and Wildlife Service to avoid or minimize take of migratory birds at these structures. Proceedings of the Fourth International Partners in Flight Conference: Tundra to Tropics 262-272 (Partners in Flight, US, 2009).

21. Longcore, T. et al. An estimate of mortality at communication towers in the United States and Canada. PLoS one 7, e34025 (2012).

22. Pullin, A. S. \& Stewart, G. B. Guidelines for systematic review in conservation and environmental management. Conserv. Biol. 20, 1647-1656 (2006).

23. Loss, S. R., Will, T. \& Marra, P. P. Direct human-caused mortality of birds: improving quantification of magnitude and assessment of population impact. Front. Ecol. Environ. 20, 357-364 (2012).

24. Henderson, R. W. Consequences of predator introductions and habitat destruction on amphibians and reptiles in the Post-Columbus West Indies. Caribb. J. Sci. 28, 1-10 (1992).

25. Nilsson, N. N. The role of the domestic cat in relation to game birds in the Willamette Valley, Oregon. Thesis (Oregon State College, 1940).

26. Llewellyn, L. L. \& Uhler, F. M. The foods of fur animals of the Patuxent Research Refuge, Maryland. Am. Midl. Nat. 48, 193-203 (1952).

27. Parmalee, P. W. Food habits of the feral house cat in east-central Texas. J. Wildl. Manage 17, 375-376 (1953).

28. Hubbs, E. L. Food habits of feral house cats in the Sacramento Valley. Calif. Fish Game 37, 177-189 (1951).

29. Jackson, W. B. Food habits of Baltimore, Maryland, cats in relation to rat populations. J. Mammal. 32, 458-461 (1951).

30. Errington, P. L. Notes on food habits of southern Wisconsin house cats. J. Mammal. 17, 64-65 (1936).

31. Kays, R. W. \& DeWan, A. A. Ecological impact of inside/outside house cats around a suburban nature preserve. Anim. Conserv. 7, 273-283 (2004).

32. Blancher, P. J. et al. Guide to the partners in flight population estimates database version: North American Landbird Conservation Plan 2004, Tech. Series No 5 (Partners in Flight, US, 2007).

33. Barratt, D. G. Predation by house cats, Felis catus (L.), in Canberra, Australia. I: prey composition and preference. Wildl. Res. 24, 263-277 (1997).

34. Barratt, D. G. Predation by house cats, Felis catus (L.), in Canberra, Australia. II: Factors affecting the amount of prey caught and estimates of the impact on wildlife. Wildl. Res. 25, 475-487 (1998). 
35. Liberg, O. Food habits and prey impact by feral and house-based domestic cats in a rural area in southern Sweden. J. Mammal. 65, 424-432 (1984).

36. Jones, E. Ecology of the feral cat, Felis catus (L.), (Carnivora:Felidae) on Macquarie Island. Aust. Wildl. Res. 4, 249-262 (1977).

37. Bramley, G. N. A small predator removal experiment to protect North Island Weka (Gallirallus australis greyi) and the case for single-subject approaches in determining agents of decline. NZ J. Ecol. 20, 37-43 (1996).

38. Bonnaud, E. et al. The diet of feral cats on islands: a review and a call for more studies. Biol. Conserv. 13, 581-603 (2011).

39. Tschanz, B., Hegglin, D., Gloor, S. \& Bontadina, F. Hunters and non-hunters: skewed predation rate by domestic cats in a rural village. Eur. J. Wildl. Res. 57, 597-602 (2011).

40. Fiore, C. A. Domestic cat (Felis catus) predation of birds in an urban environment. Thesis (Wichita State University, 2000).

41. Blancher, P. J. Estimated number of birds killed by house cats (Felis catus) in Canada. (Avian Conservation and Ecology, in press).

\section{Acknowledgements}

S.R.L. was supported by a postdoctoral fellowship funded by the US Fish and Wildlife Service through the Smithsonian Conservation Biology Institute's Postdoctoral Fellow ship programme. P. Blancher provided insight for development of the model of cat predation magnitude, and R. Kays, C. Lepczyk and Y. van Heezik provided raw data from their publications. C. Machtans facilitated data sharing, and participants in the 2011
Society of Canadian Ornithologists' anthropogenic mortality of birds symposium provided context and perspectives. C. Lepczyk and P. Blancher provided comments on the manuscript. The findings and conclusions in this article are those of the authors and do not necessarily represent the views of the Smithsonian or US Fish and Wildlife Service. All data used for this analysis is available in the Supplementary Materials.

\section{Author contributions}

S.R.L. designed the study, collected and analysed data, and wrote the paper. T.W. and P.P.M. designed the study and contributed to paper revisions. All authors discussed the results and commented on the manuscript.

\section{Additional information}

Supplementary Information accompanies this paper on http://www.nature.com/ naturecommunications

Competing financial interests: The authors claim no competing financial interests associated with this paper.

Reprints and permission information is available online at http://npg.nature.com/ reprintsandpermissions/

How to cite this article: Loss S.R. et al. The impact of free-ranging domestic cats on wildlife of the United States. Nat. Commun. 4:1396 doi: 10.1038/ncomms2380 (2012). 


\title{
Corrigendum: The impact of free-ranging domestic cats on wildlife of the United States
}

\author{
Scott R. Loss, Tom Will \& Peter P. Marra
}

Nature Communications 4:1396 doi:10.1038/ncomms2380 (2013); Published 29 Jan 2013; Updated 12 Dec 2013

The original version of this Article contained incorrect estimates of the number of animals killed by free-ranging domestic cats, which were based on $90 \%$ confidence levels rather than the stated $95 \%$ confidence levels. Reanalysis of the original data after publication using 95\% confidence levels resulted in larger ranges for the estimated number of animals killed. For example, in the fifth sentence of the Abstract, the number of birds killed by free-ranging domestic cats required revision from 1.4-3.7 billion to $1.3-4.0$ billion and the number of mammals killed by free-ranging domestic cats required revision from 6.9-20.7 billion to 6.3-22.3 billion. The estimates have now been corrected throughout the PDF and HTML versions of the Article.

Unrelated to the changes above, four estimates of cat predation rates on wildlife from temperate zone studies in Supplementary Table S1 were based on partial year values that had not been adjusted to year-round estimates. The values have now been revised in Supplementary Table S1.

The original conclusions are not affected by any of the above changes. 